\title{
Effects of a Thermistor String Mounted between the Acoustic Beams of an Acoustic Doppler Current Profiler
}

\author{
FRIEDRICH SCHOTT \\ Rosenstiel School of Marine and Atmospheric Science, University of Miami, Miami, Florida
}

31 December 1986 and 21 July 1987

\begin{abstract}
A useful extension of upward-looking buoy-mounted acoustic Doppler current profilers (ADCPs), in particular for studying surface-mixed-layer dynamics from underneath, would be to combine them with a thermistor cable for obtaining simultaneous current and temperature profiles. Measurements with an ADCP, operating at 150 $\mathrm{kHz}$ and sampling at $2.2 \mathrm{~m}$ vertical bin lengths, were carried out from a barge moored in Lake San Vincente, California, over a water depth of $45 \mathrm{~m}$, to investigate the effects of a thermistor string running along the axis of the instrument between the acoustic beams and held by a small auxiliary float. The biasing effect of the thermistor string itself was only of order $1 \%$. Doppler biasing of the horizontal currents by two different kinds of floats (one a cylindrical hard foam float with low acoustical reflectivity, the other a hollow sphere) was investigated. The floats were suspended at 15 and $30 \mathrm{~m}$. For floats at $30 \mathrm{~m}$, evidence was not conclusive because of apparent interference by other reflectors. The cylindrical foam float at $15 \mathrm{~m}$ caused no measurable Doppler biasing, while the effect of the hollow sphere was of order $40 \%$ in the depth bin around $15 \mathrm{~m}$.
\end{abstract}

\section{Introduction}

Self-contained acoustic Doppler current profilers (ADCPs), mounted in the top buoy of a subsurface mooring and looking upward, are becoming a standard tool in oceanography. They have been shown to provide good quality current profiles over ranges up to several hundred meters, even when deployed in strong boundary currents with significant mooring motion (Schott, 1986; Schott and Johns, 1987; Johns, 1988).

A potentially ideal application of upward-looking ADCPs is to deploy them underneath mixed layers with strong wind forcing (e.g., the Arabian Sea during the monsoon) looking upward and measuring current profiles close to the surface while not being exposed themselves to orbital wave motions or fouling effects. However, a shortcoming of this measurement technique in such an application is that one does not obtain a density or at least a temperature profile at the same time. A second mooring is needed, with a thermistor string or shipboard measurements nearby, to complement the ADCP current profiles.

The purpose of the test measurements reported here was to find out whether it would be possible to mount a thermistor string along the axis of an upward-looking $A D C P$, held vertically by a float above, without biasing the Doppler currents beyond an acceptable amount. If that would be possible the profiler could sit on top of

Corresponding author address: Dr. Friedrich Schott, Rosenstiel School of Marine and Atmospheric Science, University of Miami, Miami, FL 33149. the mooring underneath the near-surface thermocline and measure the temperature and current profiles simultaneously (Fig. 1a). The small float holding the thermistor string in that case would only have to reach up well into the homogeneous surface-mixed layer, but would not have to be exposed to wave effects close to the surface.

These tests were carried out in cooperation with RD Instruments, the manufacturer of the ADCP, on $2 \mathrm{OC}$ tober 1986 in Lake San Vincente, an artificial water reservoir near San Diego. A barge is moored on a side arm of the lake, where the water depth was about 45 $\mathrm{m}$ and the side walls of the canyon about $150 \mathrm{~m}$ away at water level. The barge has tracks along its axis on which a cart can be moved back and forth. The ADCP, operating at $150 \mathrm{kHz}$ and $20^{\circ}$ beam angle, was mounted on the cart looking downward with the transducer about $0.5 \mathrm{~m}$ below the surface; its axis orientation could be adjusted to make a single beam point downward (Fig. 1b). The track length where the cart could move freely over the water was about $5 \mathrm{~m}$.

We wanted to investigate the effect of the thermistor cable itself (Aanderaa), which has about $8 \mathrm{~mm}$ diameter and thermistors every $15 \mathrm{~m}$; and also the effect of two types of floats to be used for buoyancy. In this downward-looking application, a weight was attached below the float. We simulated the inverse of a mooring to be used later with an upward-looking ADCP on top (Fig. 1b). One float was a cylindrical hard foam float of 30 $\mathrm{cm}$ diameter and $60-\mathrm{cm}$ length; this type of float was selected to investigate whether it would be advantageous to use a long spar-buoy type float with a small 


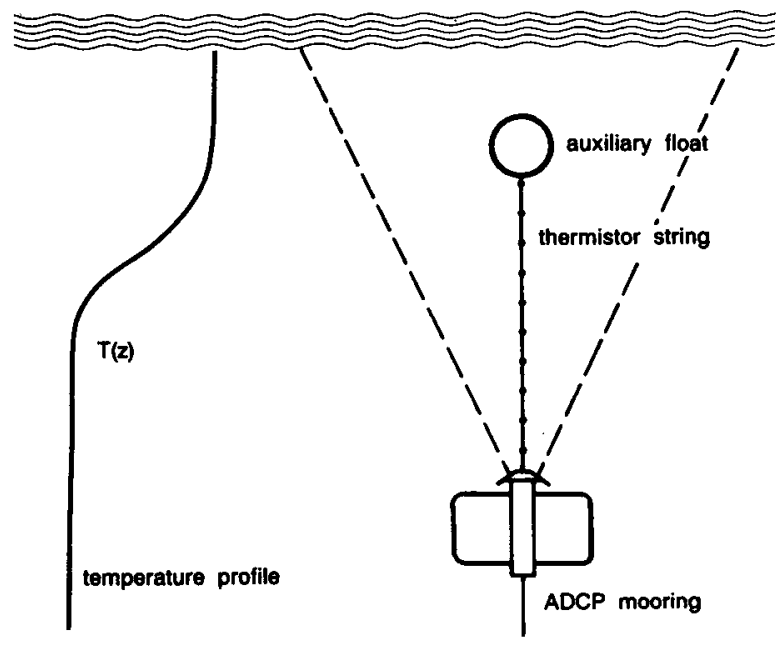

FIG. 1a. Schematic diagram of buoy-mounted upward-looking acoustic Doppler current profiler (ADCP) underneath the surface mixed layer, with a thermistor string launched between its acoustic beams to measure the temperature profile (left-hand side) from the thermocline into the mixed layer simultaneously with the Doppler currents.

cross section pointing towards the transducer. The second float was a hollow sphere of $60-\mathrm{cm}$ diameter, made of plastic with a buoyancy of about $50 \mathrm{kp}$.

Although in oceanic applications larger buoyancy floats might be needed, they would be $100 \mathrm{~m}$ or more away from the transducer, whereas here these bias effects were measured at 15 and $30 \mathrm{~m}$ distance from the transducer. Hence, the effects of floats should be over- emphasized in our experiment, compared to their real world application.

Acoustic interference could be a problem from the anchor cables, which are about $5 \mathrm{~cm}$ thick and run slantwise downward from the four corners of the barge. Another probable source of acoustical noise is fish; fairly large catfish were constantly in view underneath the barge.

\section{Observations}

The $150-\mathrm{kHz}$ Doppler profiler (which actually operates at $153.6 \mathrm{kHz}$ ) has a four-beam Janus configuration. In our application, beams 3 and 4 were oriented opposite to each other along the axis of the cart, and beams 1 and 2 normal to the axis. The acoustical measurements were performed at a bin length along the beam of $2.31 \mathrm{~m}$, i.e. vertical bin length (with instrument axis pointing downward) of $2.17 \mathrm{~m}$; ping rate of $4 \mathrm{~Hz}$ and ensemble averaging of 100 pings, i.e. ensemble averaging time of 25 seconds. The transducer surface was at about $0.2 \mathrm{~m}$ depth and the blanking period 1.1 $\mathrm{m}$, i.e., bin 1 began at a depth of $1.3 \mathrm{~m}$. We recorded the echo amplitudes of the four beams, the Doppler velocities of the four beams and the vector-averaged vertical and horizontal current components; the latter oriented along and normal to the axis of the cart.

Doppler bias will occur in a depth bin if an acoustic obstacle (i.e. our cable and/or float) which does not move relative to the transducer is located within the moving water. The effect will be biasing toward lower velocities, its magnitude depending on the weight of side view

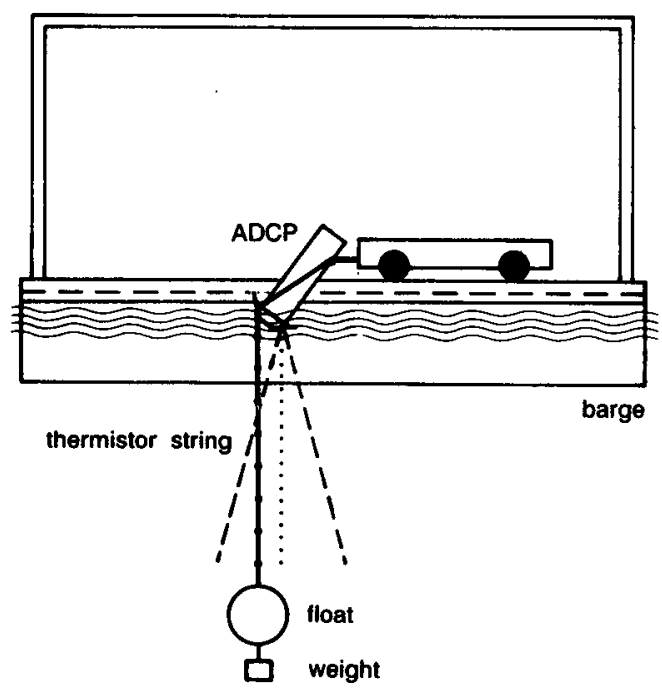

front view

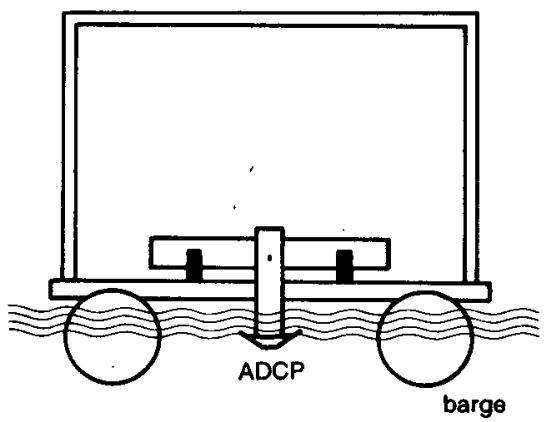

FIG. 1b. Test arrangement on the barge on Lake San Vincente. The ADCP is mounted on a cart which can be moved along a track; the instrument can be tilted such that either a single beam (as shown in side view) or the instrument axis points downward. The thermistor string with float is suspended in the inverse situation of Fig. 1a, with a weight below the buoy, to test the effect of thermistor string and float on Doppler current signals. 
the scattering by the obstacle relative to that by the moving scatterers (small particles, zooplankton). This biasing effect, for instance, is drastically observed when profiling up to the surface from underneath. In that case, the strong direct return echoes, which travel straight up to the surface and back and are received through sidelobes of the beam patterns, superimpose on the echoes coming back along the inclined beam and bias the horizontal Doppler velocities towards zero velocities (Schott, 1986). An added effect is the increase in current in the boundary layer of the nonmoving obstacle itself, which will reduce the bias towards low velocities.

The following series of observations were carried out:

1) Point one beam vertically; lower and raise thermistor string with a small weight repeatedly at speeds of $1-2 \mathrm{~m} \mathrm{~s}^{-1}$. This is a measurement of the maximum effect of the thermistor string, i.e. when it is oriented parallel to the flow of water. In further course of experiment, point instrument axis vertical.

2) Move cart back and forth repeatedly without thermistor cable for reference measurements. Suspend thermistor string vertically along instrument axis and attach

3) round float at $15 \mathrm{~m}$

4) foam float at $15 \mathrm{~m}$

5) round float at $30 \mathrm{~m}$

6) foam float at $30 \mathrm{~m}$ to measure the effects of both floats on echo amplitude and look for Doppler biasing.

The random error $V_{E}$ due to acoustical noise is given by the empirical formula (RD Instruments, 1985):

where

$$
V_{E}=\frac{18000}{F R \cos (i)}(B N)^{-1 / 2}
$$

$F$ transducer frequency $(\mathrm{kHz})$

$R$ bin length along the beam (m)

$i$ angle between velocity component and beam

$B$ number of acoustic beams

$N$ number of pings.

For our parameters of $N=100, R=2.31 \mathrm{~m}$ :

(i) for a single beam and the radial velocity component (i.e., case 1) $V_{E}=5.1 \mathrm{~cm} \mathrm{~s}^{-1}$;

(ii) for the beam instrument axis pointing vertically and 4 beams (i.e., cases $2-6$ ):

horizontal component error velocity $V_{E H}=7.4 \mathrm{~cm} \mathrm{~s}^{-1}$

vertical component error velocity $V_{E V}=2.7 \mathrm{~cm} \mathrm{~s}^{-1}$.

For each of the cases 2-6 the cart was moved back and forth, 5-10 times in each direction. Since this was an improvised experiment we did not have a motorized drive system set up to move the cart at a controlled speed. Instead we pushed it along the track by hand such that it passed the available track length of $5 \mathrm{~m}$ in about the ensemble sampling interval of 25 seconds, i.e. at a speed of about $20 \mathrm{~cm} \mathrm{~s}^{-1}$. In the analysis we averaged the repetitive cases in order to reduce the standard deviations.

\section{Results}

\section{a. Effect of thermistor string only}

This case is the most drastic way the thermistor string can affect the data, i.e., if the string is oriented such that it is directly within a beam and the biasing Doppler effect is applied directly along the beam axis. The iron weight at the end of the string will also cause an effect as it travels up and down through the bins, but at $2 \mathrm{~m}$ $\mathrm{s}^{-1}$ vertical speed it would only affect four pings out of an ensemble of 100 for each bin.

We lowered the thermistor string at approximately $2 \mathrm{~m} \mathrm{~s}^{-1}$ and raised it at about $1-1.5 \mathrm{~m} \mathrm{~s}^{-1}$ while beam 3 was pointing downward and data collection carried out at $4 \mathrm{~Hz}$. Approximately one ensemble of 100 pings was collected during the lowering and more during the raising.

Table 1 shows the Doppler velocities for the four individual beams and the velocity along the axis from the combination of all beams. Data are averaged over four ensembles of each case and the top five bins. The acoustical amplitude for this case is $1.1 \mathrm{~cm} \mathrm{~s}^{-1}$, based on the discussion in section 2.

The first line shows the results for four reference ensembles, when the thermistor string was suspended but not moving; Doppler velocities along all beams are close to zero. The second line shows the effect of lowering the cable at about $2 \mathrm{~m} \mathrm{~s}^{-1}$. The means of beams 1,2 , and 4 are now zero or slightly negative (but none of these changes is significant) while beam 3 shows a mean of $2.3 \pm 0.6 \mathrm{~cm} \mathrm{~s}^{-1}$ downward. This would suggest that the effect of the vertically moving thermistor string shows as an about $1 \%$ biasing of the Doppler current along the beam. On the other hand, when the thermistor string was pulled upwards (line 3 in Table 1) no consistent positive biasing can be detected when averaging over four profiles. The average effect in that case is still negative for beam 3 but not significant at $-0.6 \pm 0.6 \mathrm{~cm} \mathrm{~s}^{-1}$ and the average velocity along the instrument axis is $0.2 \pm 0.5 \mathrm{~cm} \mathrm{~s}^{-1}$.

One possible explanation for the difference in Doppler velocity between downward and upward

TABLE 1. Doppler velocities ( $\mathrm{cm} \mathrm{s}^{-1}$ ) along the four beams and along instrument axis $(V Z)$, averaged over four profiles and top 5 bins while thermistor chain was at rest, lowered, or raised and beam 3 was pointing vertically downwards. Standard deviations are those of the mean.

\begin{tabular}{lccrrr}
\hline \hline & \multicolumn{5}{c}{ Beam } \\
\cline { 2 - 6 } & \multicolumn{1}{c}{1} & 2 & \multicolumn{1}{c}{3} & \multicolumn{1}{c}{4} & \multicolumn{1}{c}{$V Z$} \\
\hline At rest & $-.4 \pm .4$ & $.8 \pm .4$ & $-0.3 \pm 0.6$ & $.2 \pm .4$ & $0.5 \pm 0.8$ \\
Chain down & $-.2 \pm .3$ & $.0 \pm .5$ & $-2.3 \pm 0.6$ & $-.3 \pm .4$ & $-2.0 \pm 0.9$ \\
Chain up & $-.1 \pm .4$ & $.5 \pm .4$ & $-0.6 \pm 0.6$ & $.8 \pm .4$ & $0.2 \pm 0.5$ \\
\hline
\end{tabular}


mooring cable could be that the cable, which was lined up on deck, was carrying air bubbles down into the water along its coating when lowered.

In summary, even if we assume that the downward biasing observed is real, it appears that the effect of a fixed thermistor string lying in an alongbeam flow reduces the measured velocity magnitude by less than about $1 \%$.

\section{b. Effect of floats}

Mean echo amplitudes, averaged over the four beams (instrument axis always downward) are shown in Fig. 2 for the reference case without any float or chain and for the cases of both kinds of float at 15 and at $30 \mathrm{~m}$ depth respectively. All profiles show a decrease in the first one or two bins, which was also found in some oceanic applications (e.g. Schott, 1986). Echo amplitudes increase below $40 \mathrm{~m}$, apparently due to sidelobe returns from the bottom.

Just looking at the echo amplitudes for both kinds of floats at $30 \mathrm{~m}$ (Fig. 2) one might conclude that there is a noticeable increase of echo amplitude due to the floats. However, the reference case with no chain or float and some of the measurements with floats at 15 $\mathrm{m}$ also show this increase.

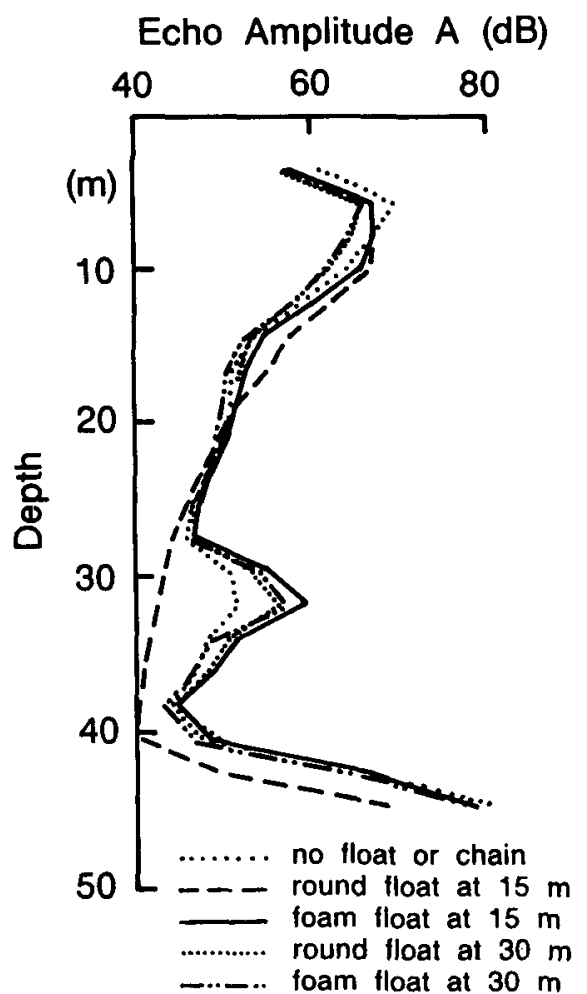

FIG. 2. Mean echo amplitudes, averaged over the four beams and different number of ensembles for reference case with no chain or float and both kinds of floats (round sphere and cylindrical hard foam) at 15 and $30 \mathrm{~m}$, respectively.
In Fig. 3a we averaged again over the cases of both kinds of floats at 15 and at $30 \mathrm{~m}$, and both profiles are now fairly similar. These averages are over 28 ensembles for the $15 \mathrm{~m}$ level floats and 22 ensembles for the $30 \mathrm{~m}$ floats. Both profiles show the excursion at about $30 \mathrm{~m}$. The profile for floats at $30 \mathrm{~m}$ has slightly higher echo amplitudes around that depth; the profile for floats at $15 \mathrm{~m}$ has slightly higher amplitudes above $18 \mathrm{~m}$ but not just at $\sim 15 \mathrm{~m}$.

The secondary echo amplitude maximum at $30 \mathrm{~m}$ is a puzzling effect. It occurred (i) for the reference case, whether or not the cart was moving; (ii) for both kinds of floats at $30 \mathrm{~m}$ it occurred when the cart was moving but not when it was at rest; (iii) for the foam float at $15 \mathrm{~m}$, the same situation as in (ii); but (iv) for the round float at $15 \mathrm{~m}$ it did not occur. The maximum occurred in all four beams. The largest signal was always beam 1 , i.e. the one pointing to the right for the cart moving outward. One possibility for the effect would be reflections from the anchor cables during. some part of the path along the track, probably on the outer part of the track. This is supported by the fact that measurements at rest which were made with the cart at the center of the barge were not biased by this effect except for the reference case (i) where the cart was stopped at the outer end of the track during the at-rest recording period. Still, the question remains why does the effect not occur for the round float at $15 \mathrm{~m}$.

Another possibility could be fish concentrations at about $30 \mathrm{~m}$ depth; fish were observed visually under the barge. There is also the possibility of structures or trees on the ground of the sunken valley interfering in that depth range. The effect at $30 \mathrm{~m}$ does not, however, appear to be related to the thermal structure because from earlier BT profiles ( $R$. Merewether, personal communication) it is known that the water below about $20 \mathrm{~m}$ depth is homogeneous throughout the year.

Moving the profiler with thermistor string and float attached through the water should yield a constant speed profile in the beam pair parallel to the track corresponding to the reverse of the speed of the profiler, assuming negligible depth dependence of currents in the lake. The effect to be looked for is biasing by the floats toward lower Doppler currents in that part of the profile. For each of the two levels, 15 and $30 \mathrm{~m}$, and the two types of floats, hollow sphere and cylindrical foam float, Doppler currents of the component along the axis of the barge were averaged over a number of ensembles at rest and for both directions of the moving cart.

Table 2 shows the results for both floats at $15 \mathrm{~m}$ depth. Also shown for each float case are the speeds averaged for both directions of the moving cart (inverting the signs of measurements for the cart moving out), which make a total of 10 cases for the round and 18 for the cylindrical hard foam float. Averages are taken over two adjacent bins, beginning with bin 2; results from bin 7 in which the float was located are 


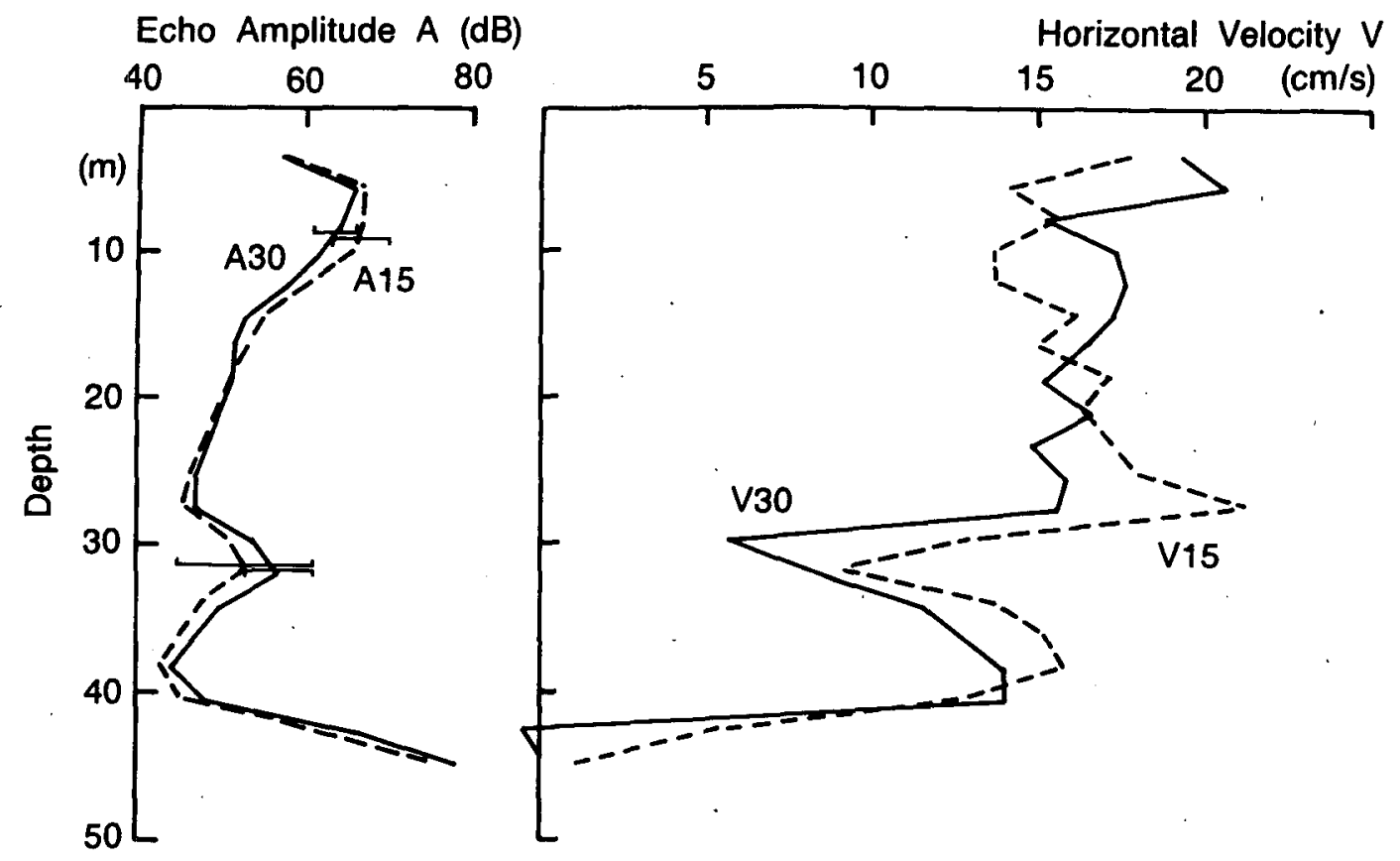

FIG. 3. (a) Mean echo amplitudes averaged for ensembles with both kinds of floats at $15 \mathrm{~m}$ depth (dashed) and at $30 \mathrm{~m}$ depth (solid). Standard deviations are shown at the $15 \mathrm{~m}$ and $30 \mathrm{~m}$ levels. (b) As in (a) except for Doppler currents.

also shown individually. There appears to be a biasing effect by the round float in bin 7 , where the float was located, 14.3-16.5 $\mathrm{m}$ depth, in comparison to the currents averaged over depth ranges above and below. The mean current (averaged over the ten cases of the cart moving either way) for the depth range $3.5-12.2 \mathrm{~m}$ above the float and 16.5-25.2 m below it (which is still undisturbed by the funny effects of the $30 \mathrm{~m}$ level) is $15.4 \pm 2.3 \mathrm{~cm} \mathrm{~s}^{-1}$ (Table 2, last line); while the current in bin 7 was $9.1 \pm 2.7 \mathrm{~cm} \mathrm{~s}^{-1}$. For the cylindrical hard foam float no such biasing in bin 7 is detectable. The biasing can even be detected for the at-rest case of the sphere where the mean is $7.8 \pm 2.6 \mathrm{~cm} \mathrm{~s}^{-1}$, while the bin 7 current, at $3.6 \pm 2.8 \mathrm{~cm} \mathrm{~s}^{-1}$, is the smallest of all bins above the near bottom range. A somewhat puzzling result is that the average current in bins 4-5, depth range $7.8-12.2 \mathrm{~m}$ is also lower than at bins $2-3$ and 8-11 (Table 2). It is unlikely that this was genuine negative current shear above $12 \mathrm{~m}$ because the at rest measurements showed a fairly constant current profile of

TABLE 2. Doppler currents for floats at $15 \mathrm{~m}$ depth.

\begin{tabular}{|c|c|c|c|c|c|c|c|c|c|c|}
\hline \multicolumn{2}{|c|}{ Averages over } & \multicolumn{4}{|c|}{ Round float cart moving } & & & & & \multirow{3}{*}{$\begin{array}{c}\text { Average } \\
\text { both } \\
\text { floats }\end{array}$} \\
\hline & denth & \multirow{2}{*}{ At rest } & \multirow[b]{2}{*}{ Out } & \multirow[b]{2}{*}{ In } & \multirow{2}{*}{$\begin{array}{l}\text { Average } \\
(\mathrm{cm} / \mathrm{s})\end{array}$} & \multicolumn{4}{|c|}{ Foam float cart moving } & \\
\hline bin & (m) & & & & & At rest & Out & In & Average & \\
\hline $2-3$ & $3.5-7.8$ & $6.0 \pm 1.9$ & $-9.3 \pm 2.2$ & $21.6 \pm 2.6$ & $15.5 \pm 2.2$ & $-3.1 \pm 2.1$ & $-10.3 \pm 2.0$ & $18.7 \pm 2.5$ & $14.5 \pm 1.7$ & $14.8 \pm 1.3$ \\
\hline $4-5$ & $7.8-12.2$ & $5.3 \pm 3.4$ & $-6.3 \pm 1.4$ & $17.3 \pm 2.5$ & $11.8 \pm 1.9$ & $2.6 \pm 1.8$ & $-11.2 \pm 1,8$ & $18.3 \pm 1.5$ & $14.8 \pm 1.3$ & $13.7 \pm 1.1$ \\
\hline $6-7$ & $12.2-16.5$ & $5.7 \pm 3.2$ & $-8.4 \pm 2.3$ & $15.8 \pm 2.9$ & $12.1 \pm 2.0$ & $3.8 \pm 2.7$ & $-14.8 \pm 2.0$ & $20.1 \pm 2.0$ & $17.4 \pm 1.4$ & $15.5 \pm 1.2$ \\
\hline 7 & $14.3-16.5$ & $3.6 \pm 2.8$ & $-5.2 \pm 3.1$ & $12.9 \pm 4.0$ & $9.1 \pm 2.7$ & $7.0 \pm 1.9$ & $-17.3 \pm 3.2$ & $19.1 \pm 2.5$ & $18.2 \pm 2.0$ & $14.9 \pm 1.8$ \\
\hline $8-9$ & $16.5-20.8$ & $7.9 \pm 2.7$ & $-10.3 \pm 3.2$ & $22.0 \pm 2.7$ & $16.2 \pm 2.4$ & $2.0 \pm 1.8$ & $-16.7 \pm 2.2$ & $17.5 \pm 1.8$ & $17.1 \pm 1.4$ & $16.8 \pm 1.2$ \\
\hline $10-11$ & $20.8-25.2$ & $12.0 \pm 2.5$ & $-8.5 \pm 3.2$ & $27.8 \pm 2.6$ & $18.2 \pm 3.0$ & $1.9 \pm 1.8$ & $-15.5 \pm 2.1$ & $18.8 \pm 2.4$ & $17.1 \pm 1.6$ & $17.4 \pm 1.5$ \\
\hline $12-13$ & $25.2-29.5$ & $5.6 \pm 2.1$ & $-18.8 \pm 2.3$ & $24.6 \pm 2.1$ & $21.7 \pm 1.7$ & $3.5 \pm 1.9$ & $-11.8 \pm 3.3$ & $17.6 \pm 2.2$ & $14.7 \pm 2.0$ & $17.2 \pm 1.5$ \\
\hline $14-15$ & $29.5-33.9$ & $3.8 \pm 2.2$ & $-12.3 \pm 2.8$ & $17.3 \pm 2.7$ & $14.8 \pm 2.0$ & $1.0 \pm 2.0$ & $-7.1 \pm 1.7$ & $11.7 \pm 2.5$ & $9.4 \pm 1.6$ & $11.3 \pm 1.3$ \\
\hline $16-17$ & $33.9-38.2$ & $2.2 \pm 2.2$ & $-16.0 \pm 2.0$ & $15.6 \pm 2.6$ & $15.8 \pm 1.6$ & $-3.5 \pm 2.3$ & $-10.6 \pm 1.8$ & $19.8 \pm 2.2$ & $15.2 \pm 1.6$ & $15.4 \pm 1.2$ \\
\hline $2-5$ & $3.5-12.1$ & . & & & & & & & & \\
\hline and & and & & & & & & & & & \\
\hline $8-11$ & $16.5-25.2$ & $7.8 \pm 2.6$ & $-8.6 \pm 1.5$ & $22.2 \pm 3.7$ & $15.4 \pm 1.9$ & $.9 \pm 2.2$ & $-13.4 \pm 2.7$ & $18.3 \pm .5$ & $15.9 \pm 1.2$ & $15.7 \pm 1.5$ \\
\hline \multicolumn{11}{|l|}{$\begin{array}{c}\text { Number of } \\
\text { profiles }\end{array}$} \\
\hline averaged & & 6 & 5 & 5 & 10 & 6 & 9 & 9 & 18 & 28 \\
\hline
\end{tabular}


between 5.3 and $6.0 \mathrm{~cm} \mathrm{~s}^{-1}$ in bins 2-5 (Table 2), and the moving cart measurements were made only a few minutes later; neither did the barge change its orientation noticeably, and movements of the barge itself on its anchor cables would add only depth-independent currents. Sidelobe effects through vertically traveling returns from the float could also not cause an early arrival in bin 5 for the float at $15 \mathrm{~m}$. On the other hand, the echo amplitude for the round float at $15 \mathrm{~m}$ corroborates this result: it is larger, although not signifcantly, in some range above $15 \mathrm{~m}$ (Fig. 3a).

No Doppler current biasing can be detected for the cylindrical foam float (Table 2) and only small echo amplitude increases compared to the cases of no float at $15 \mathrm{~m}$ (Fig. 2). The profile for the currents averaged over all 28 ensembles of both floats at $15 \mathrm{~m}$ (Table 2) is shown in Fig. 3b. The Doppler biasing toward zero velocities is obvious near the bottom where the echo amplitude increases due to direct returns, 'traveling vertically through the water. While the depth range between 10 and $15 \mathrm{~m}$ shows moderate biasing towards lower velocities when averaged over both $15 \mathrm{~m}$ floats, there is a large anomaly between 25 and $35 \mathrm{~m}$, corresponding to the anomalous increase in echo amplitude in that depth range (Figs. 1 and 3a).

Because of the unexplained effects at $30 \mathrm{~m}$ depth from sources other than our floats, the results equivalent to Table 2 but with both kinds of floats at $30 \mathrm{~m}$ are not presented. Comparing the echo amplitude profiles in Fig. 3a for floats at $15 \mathrm{~m}$ and at $30 \mathrm{~m}$ shows higher echo amplitude at $\sim 30 \mathrm{~m}$ for floats at $30 \mathrm{~m}$, superimposed on the existing anomaly at that depth while Doppler currents are biased low at that depth for $30 \mathrm{~m}$ floats compared to $15 \mathrm{~m}$ floats.

\section{Summary and conclusions}

We attempted, in an experiment in Lake San Vincente, California, to find out whether it is feasible in combining an upward-looking acoustic Doppler current profiler (ADCP) with a thermistor string held upward by an ancillary float to obtain simultaneous current and temperature profiles.

The experiment was carried out on a barge in water depth of approximately $45 \mathrm{~m}$. The ADCP was mounted on a cart that could be moved on tracks along the axis of the barge with the transducer in the water, pointing downwards.

First we tested the effect of the thermistor cable itself, by lowering or raising it directly within a vertically pointing acoustic beam. This effect was found to be small and to cause a biasing effect on the vertical currents of less than $1 \%$.

Then we tested the effects of two different floats holding the thermistor string; one was a hollow sphere of $60-\mathrm{cm}$ diameter and the second a cylindrical hard foam float of $30-\mathrm{cm}$ diameter and $60 \mathrm{~cm}$ length.
Measurements with both types of floats suspended vertically by the thermistor string to depths of 15 and $30 \mathrm{~m}$ were carried out in which the entire system was moved horizontally through the water at speeds of 15$20 \mathrm{~cm} \mathrm{~s}^{-1}$, during which time one ensemble of 100 pings was recorded.

For the depth range around $30 \mathrm{~m}$ anomalies in echo amplitudes and Doppler currents occurred independently of the floats, probable causes being either one or a combination of interference of sidelobe echoes from the anchor cables of the barge, from trees or structures on the bottom of the sunken valley, or from fish concentrations. Yet, a difference was also detectable at that depth, when floats were at $30 \mathrm{~m}$, resulting in increased echo amplitude and reduced Doppler currents.

For floats at $15 \mathrm{~m}$, the results were cleaner because lack of obvious interference from other reflectors. While the cylindrical hard foam float showed no measurable Doppler current bias, the hollow sphere had a biasing effect of order $40 \%$ on the currents of the 2.2 $\mathrm{m}$ long depth bin in which the sphere was located. The poorer performance of the hollow sphere, compared to the spar-buoy type foam float, is to be expected. Not only was the cross section of the sphere four times that of the foam float, but the foam float has much better acoustic characteristics for this kind of application. It has a density of $0.51 \mathrm{~g} \mathrm{~cm}^{-3}$ and a sound velocity about twice that of seawater (personal communication by the manufacturer) which makes its acoustical impedance very close to that of seawater.

Bins above the $15 \mathrm{~m}$ level appeared to be affected by the bias both through increased echo amplitude and reduced Doppler currents, but this effect was not significant and could be spurious; no apparent reason for its existence could be found.

It can be concluded that a thermistor string held by a hard foam float between the beams of an upwardlooking ADCP should not cause problems in the acoustic Doppler currents measured.

Acknowledgments. I thank Fran Rowe and Ray Merewether of RD Instruments and Phil Bedard of RSMAS for helping with the experiment; Rainer Zantopp for his part in the data analysis; and Bill Johns for helpful discussions. The work was supported by ONR under Contract N00014-85-C-0020 and by NOAA under Contract NA85-WC-4-06134.

\section{REFERENCES}

Johns, W., 1988: Near-surface current measurements in the Gulf Stream using an upward-looking acoustic Doppler current profiler. J. Atmos. Oceanic Technol., (in press).

RD Instruments, 1985: Acoustic Doppler current profilers. Techn. Data Sheet.

Schott, F., 1986: Medium-range vertical acoustic Doppler current profiling from submerged buoys. Deep-Sea Res., 33, 1279-1292. , and W. Johns, 1987: Half-year long measurements with a buoymounted acoustic Doppler current profiler in the Somali Current. J. Geophys. Res., 92, 5169-5176. 